\title{
Avaliação de usabilidade do SofEMN: Software de apoio ao Ensino de Métodos Numéricos
}

\author{
Alice Fonseca Finger ${ }^{1}$, Aline Brum Loreto ${ }^{2}$, \\ Ana Luisa Soubhia ${ }^{2}$, Arthur Cordeiro Andrade ${ }^{2}$ \\ ${ }^{1}$ Universidade Federal do Pampa (UNIPAMPA) \\ Av. Tiarajú, 810, Ibirapuitã - Alegrete, RS - Brasil \\ ${ }^{2}$ Universidade Federal de Santa Maria (UFSM), CS \\ Rod. Taufik Germano, 3013, Passo D'Areia - Cachoeira do Sul, RS - Brasil \\ alicefinger@unipampa.edu.br; \{aline.loreto, ana.soubhia\}@ufsm.br

\begin{abstract}
The goal of this paper is to show the results obtained from the application of a usability test of free mathematical software, called SofEMN (Education Software of Numerical Methods), developed in a research project by professors and students of the Federal University of Santa Maria. The test results show that students approve the initiative to use specific software for numerical methods during the teaching-learning process. Furthermore, SofEMN needs minor review, mainly in the interface, to improve the visualization of screens and buttons. In this way, this tool could contribute to the knowledge in the area.
\end{abstract}

Resumo. O objetivo desse trabalho é apresentar os resultados obtidos a partir da aplicação de um primeiro teste de usabilidade do SofEMN (Software de apoio ao Ensino de Métodos Numéricos), que consiste em um software livre matemático, idealizado durante a execução de um projeto de pesquisa desenvolvido por docentes e discentes da UFSM. Os resultados do teste mostram que os estudantes aprovam a iniciativa de utilizar um software específico para métodos numéricos durante o processo de ensino e aprendizagem. Além disso, que o SofEMN precisa de pequenos ajustes, principalmente com relação a interface, com o objetivo de melhorar a visualização das telas e botões, para que se torne uma ferramenta de auxílio para solidificar o conhecimento na área.

\section{Introdução}

Métodos Numéricos Computacionais, ou Cálculo Numérico, é uma disciplina obrigatória em diversos cursos de engenharia, que consiste em abordar conceitos sobre erros, métodos numéricos para obtenção de zeros, aproximações e interpolação de funções, além de métodos de integração numérica e de resolução de sistemas lineares e não lineares. Todos esses métodos são estudados de forma teórica e prática. Na parte prática, diversas linguagens de programação podem ser utilizadas, como por exemplo, a linguagem de programação C, a qual não proporciona ambiente matemático adequado. Salienta-se que um desafio que pode ser encontrado é com relação ao alto grau de abstração e criatividade, os quais são necessários para a obtenção de habilidade com a lógica de programação [Universidade Federal de Santa Maria-CS 2019].

Segundo [Romero 2006], a utilização de softwares matemáticos visa oportunizar a motivação e apropriação do conteúdo estudado em sala de aula. Observa-se que no atual 
contexto do ensino remoto é crescente a dependência do uso de computadores, bem como a utilização de softwares para ensino de diversas áreas do conhecimento.

O uso de softwares para auxiliar o ensino de matemática é uma prática comum entre os professores de ensino superior. Aliar o conhecimento teórico com a prática, requer que o discente dispenda horas de estudo e escritas de fórmulas em papel. Utilizar um software que, de forma automática, consiga minimizar esse esforço é de suma importância. Conciliar as aulas teóricas com ferramentas matemáticas é essencial para uma boa formação. Dessa forma, com o intuito de auxiliar o ensino e aprendizagem de métodos numéricos, torna-se importante implementar computacionalmente os métodos para resolver problemas práticos que possuem maior complexidade.

Existem softwares matemáticos que contemplam soluções por métodos numéricos, contudo, necessitam de implementações em seus ambientes. Os softwares mais conhecidos e utilizados são: MatLab desenvolvido como um laboratório para matrizes, com diversas funções numéricas, cálculos simbólicos e ferramentas de visualização. É um software proprietário [Chapra 2013]; Octave disponibiliza comandos para resolução de problemas lineares e não-lineares numericamente, cálculo aproximado de raízes de equações não-lineares, funções ordinárias, polinômios, integrais, integração numérica, equações diferenciais ordinárias e diferenciais-algébricas. [Eaton 2021]; SciLab: O software permite manipulação com matrizes, polinômios, razões de polinômios, funções de transferência, equações de estados. [Pires 2004].

Importante salientar a necessidade de um software livre que propicie constante atualização de rotinas e pacotes para completa aplicação em diferentes problemas, das diferentes áreas das engenharias. Neste contexto, SciLab e Octave não permitem tal atualização. Tendo em vista a possibilidade de desenvolver um software em uma linguagem flexível e de código aberto, é fundamental que um software de ensino possa ser constantemente atualizado para um bom uso em sala de aula.

Segundo [Almeida et al. 2018] a identificação do software mais adequado para o auxílio no processo de aprendizagem, está diretamente relacionado ao processo de avaliação do mesmo, com o objetivo de verificar pontos positivos, negativos e respectiva adequação ao ambiente em que será utilizado.

Um dos assuntos mais abordados pela comunidade científica em eventos e periódicos nacionais é a utilização de software como auxílio ao ensino e aprendizado e avaliações de sua efetividade [Borges et al. 2015]. Neste contexto, o presente artigo apresenta o SofEMN como forma de apoio ao processo educacional de métodos numéricos, a partir de uma avaliação de usabilidade quanto à facilidade de uso, utilidade, interface e ensino em sala de aula.

O artigo está organizado da seguinte forma: A Seção 2 descreve as características de desenvolvimento do SofEMN; a Seção 3 apresenta a metodologia utilizada para a aplicação do teste de usabilidade; na Seção 4 são apresentados os resultados quantitativos e qualitativos do teste e na Seção 5 destacam-se as principais considerações deste trabalho.

\section{O software SofEMN}

O SofEMN contempla em uma única plataforma os métodos numéricos estudados na disciplina de Métodos Numéricos e Computacionais da UFSM, Campus Cachoeira do Sul, 
visando ser um auxílio no ensino dos métodos, tanto como ferramenta para demonstração de resoluções de exercícios pelos docentes como para verificação de atividades pelos discentes.

Para o desenvolvimento da ferramenta foi utilizada a linguagem de programação Python, por ser de fácil entendimento para alunos de cursos de diferentes áreas e adotada como padrão no ensino da disciplina de algoritmos. A comunidade Python Language Scientific adotou a linguagem Python para diferentes propósitos porque é uma linguagem flexível e de código aberto com extensa documentação. Outras razões que justificam o grande uso são a compatibilidade multiplataforma e a ampla disponibilidade de funções. A licença do Python é administrada pela Python Software Foundation. A Figura 1 apresenta na tela inicial o menu com os métodos numéricos implementados (versão parcial).

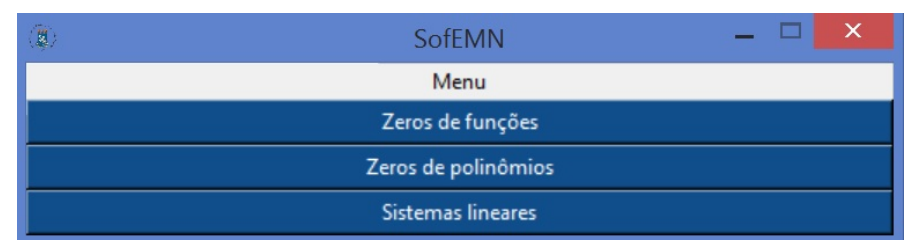

Figura 1. Menu da versão parcial do SofEMN

Os métodos numéricos implementados contemplam, segundo [Ruggiero 1996] e [Chapra 2013]: Zeros de Funções (Bissecção, Posição Falsa, Newton-Raphson e Secante), Zero de Polinômios (Birge-Vieta e Newton-Raphson), Sistemas Lineares (Cramer, Eliminação de Gauss, Fatoração LU e Fatoração Cholesky) com opções de pivoteamento parcial e total, na versão parcial, Figura 1. A versão completa possui ainda: Aproximações de Funções (Mínimos Quadrados), Interpolação (Sistemas Lineares, Lagrange e Newton) e Integração Numérica (Trapézios, 1/3 Simpson e 3/8 Simpson).

Quando o software é executado, para cada método, é solicitado que o usuário forneça informações de forma que as operações sejam realizadas. Como mostrado na Figura 2, para o cálculo de zero de funções, o usuário deve fornecer a função, o intervalo inicial $[a, b]$, o critério de parada $\varepsilon$, entre outras variáveis. Na sequência o usuário deve selecionar qual método resolverá as operações. Alguns métodos apresentam opções posteriores à obtenção do resultado, como a geração de um gráfico, possibilitando uma melhor visualização dos resultados obtidos.

Outro recurso do software é a utilização da biblioteca "mpmath", que permite o uso de precisão arbitrária, também demonstrada na Figura 2 no campo de "Precisão Dígitos". Com esse recurso, o usuário pode controlar a quantidade de dígitos significativos de precisão na apresentação dos resultados das operações realizadas pelo software. Dessa forma, é possível obter valores mais precisos do que utilizando o padrão float. Além disso, o usuário pode salvar os dados fornecidos em um arquivo, podendo recarregá-los ou repassá-los para um outro usuário, facilitando a reutilização do software.

Para melhor experiência do usuário, como o Python não utiliza de notações usuais como o "^" para potenciações e sim o '*' ou o "log"como logaritmo na base 10 e sim o " $\log 10 "$, foram desenvolvidas funções de tratamento de entrada para melhor adaptar a linguagem Python às notações usuais, como demonstrado na Figura 2. Com isso, ambas as formas de escrita podem ser utilizadas. 


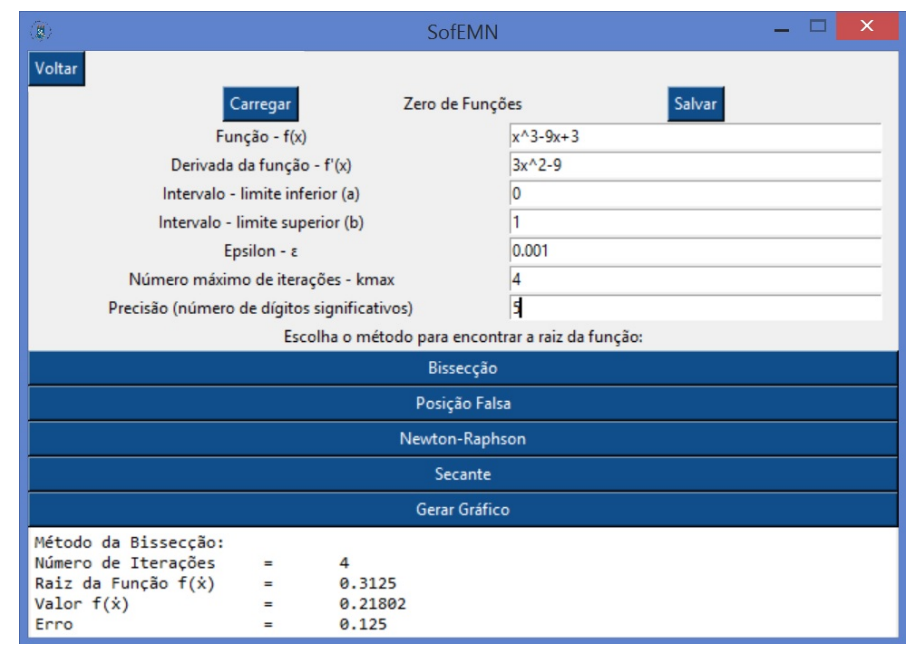

Figura 2. Exemplo de execução para o cálculo de zero de funções

\section{Planejamento e Execução do Teste de Usabilidade do SofEMN}

Um teste de usabilidade [Valentim et al. 2014] foi realizado com a versão parcial do SofEMN, antes de propô-lo nas disciplinas de métodos numéricos, com a finalidade de validar as funcionalidades já disponíveis. O objetivo do teste está descrito de acordo com GQM (Goal-Question-Metric) [Basili and Rombach 1988], que consiste em analisar a usabilidade do software com o propósito de avaliá-lo, no contexto de utilização por alunos de ensino superior, com relação à percepção sobre facilidade de uso, utilidade percebida, interface e ensino em sala de aula.

Para nortear a realização do teste, foi criado um cenário ${ }^{1}$ com passos a serem seguidos, os quais contemplam o uso de funcionalidades já vistas em sala de aula (sem uso do software) durante o andamento da disciplina de métodos numéricos. Importante salientar que, como o objetivo do teste não prevê avaliar acertos e erros de uso do software, o cenário foi descrito de maneira bem completa, a fim de garantir o uso correto das funcionalidades.

O teste foi dividido em três etapas: planejamento, execução e análise. Na etapa de planejamento foram definidas as finalidades do teste, as atividades a serem desenvolvidas, a seleção dos usuários do teste e a elaboração dos formulários que seriam utilizados durante e após o teste. A etapa de execução (aplicação do teste) ocorreu em julho de 2021, de forma remota através da plataforma Google Meet, durante as aulas da disciplina de Métodos Numéricos Computacionais da UFSM, Campus Cachoeira do Sul. O teste envolveu discentes dos cursos de Engenharias Elétrica, Mecânica, Agrícola e Transportes e Logística, divididos em três turmas, contabilizando um total de 41 participantes. Os usuários foram conduzidos pelas docentes da disciplina, as quais orientaram a seguir as atividades descritas no cenário e, depois, responderem ao questionário proposto.

A Tabela 1 contém as questões elaboradas com a finalidade de obter as informações relevantes sobre o software testado. Elas foram adaptadas ao contexto do software, com base no trabalho de [Valentim et al. 2014]. A escala Likert [Likert 1932] foi utilizada como opção de resposta às questões. As perguntas estão separadas em seções,

\footnotetext{
${ }^{1}$ https://https://bit.ly/cenario-SofEMN
} 
com a finalidade de verificar a percepção dos usuários quanto à facilidade de uso, utilidade, interface e ensino em sala de aula. Na Tabela 1, antes do nome de cada seção foi acrescentado um identificador (A, B, C, D) para otimizar a citação de cada questão no decorrer deste artigo.

Tabela 1. Questões para avaliar a percepção dos usuários do software SofEMN

\begin{tabular}{|l|l|}
\hline \multicolumn{2}{|c|}{ A - Facilidade de uso } \\
\hline $\mathbf{1}$ & Foi fácil aprender a utilizar o software \\
\hline $\mathbf{2}$ & Eu conseguia entender o que acontecia durante o uso do software \\
\hline $\mathbf{3}$ & Foi fácil ganhar habilidade de uso durante a execução das atividades no software \\
\hline $\mathbf{4}$ & É fácil de lembrar como utilizar o software \\
\hline $\mathbf{5}$ & Considero o software fácil de utilizar \\
\hline \multicolumn{2}{|c|}{ B - Utilidade do software } \\
\hline $\mathbf{1}$ & Considero o software útil para melhorar meu aprendizado \\
\hline $\mathbf{2}$ & Considero que o software melhoraria minha produtividade para realização das atividades e aprendizado \\
\hline $\mathbf{3}$ & Considero que o software facilitaria a realização das minhas atividades \\
\hline \multicolumn{3}{|c|}{ C - Interface do software } \\
\hline $\mathbf{1}$ & Considero as cores e botões do software agradáveis \\
\hline $\mathbf{2}$ & Consigo visualizar bem todos os botões e informações dentro do software \\
\hline $\mathbf{3}$ & Entendo com facilidade as palavras, nomenclaturas e ícones do software \\
\hline $\mathbf{4}$ & As imagens e ícones no software são de fácil reconhecimento \\
\hline $\mathbf{5}$ & Consigo visualizar todas as funcionalidades do software \\
\hline $\mathbf{6}$ & Consigo navegar bem por todas as telas do software \\
\hline \multicolumn{2}{|c|}{ D - Ensino em sala de aula } \\
\hline $\mathbf{1}$ & Após me acostumar com o software julgo que o software facilita o ensino e aprendizagem \\
\hline $\mathbf{2}$ & Com o software considero que as aulas ficariam mais interessantes \\
\hline $\mathbf{3}$ & Com o software eu conseguiria explorar bem os materiais disponíveis \\
\hline $\mathbf{4}$ & Com o software eu teria maior domínio sobre as atividades em sala \\
\hline $\mathbf{5}$ & Com o software o nível de aprendizagem aumentaria \\
\hline
\end{tabular}

Ao final, na etapa de análise, realizou-se a leitura e verificação dos resultados quantitativos e qualitativos, obtidos a partir do questionário aplicado. Assim, foi possível verificar a percepção dos alunos quanto ao uso do software bem como a indicação de possíveis melhorias e adequação ao uso em sala de aula.

\section{Resultados}

Como citado anteriormente, 41 discentes participaram do teste. Entretanto, dois estudantes relataram que não conseguiram utilizar o software, sendo que um deles alegou problemas com o sistema operacional Windows e o outro com o sistema operacional Linux. Dessa forma, as respostas desses participantes foram excluídas para que os resultados fossem apresentados. As análises das respostas dos questionamentos apresentados na Tabela 1 estão divididas em subseções. As três primeiras seções estão relacionadas diretamente ao software e a última envolve uma análise com relação ao ensino em sala de aula. A partir dos resultados obtidos em cada seção do questionário, gráficos foram gerados para facilitar a visualização das respostas.

\subsection{A - Facilidade de uso}

Os dados referentes à facilidade de uso servem para mostrar se os usuários tiveram facilidade para aprender e lembrar como utilizar, ganhar habilidades de uso durante a execução das tarefas, se entendiam o que acontecia durante a utilização do software e se o consideram fácil de utilizar. A Figura 3 apresenta os resultados.

De acordo com a Figura 3, a maioria dos participantes, $89.7 \%$ (35), concordou totalmente ou parcialmente que. Além disso, $92.3 \%$ (36) conseguiram entender foi fácil 


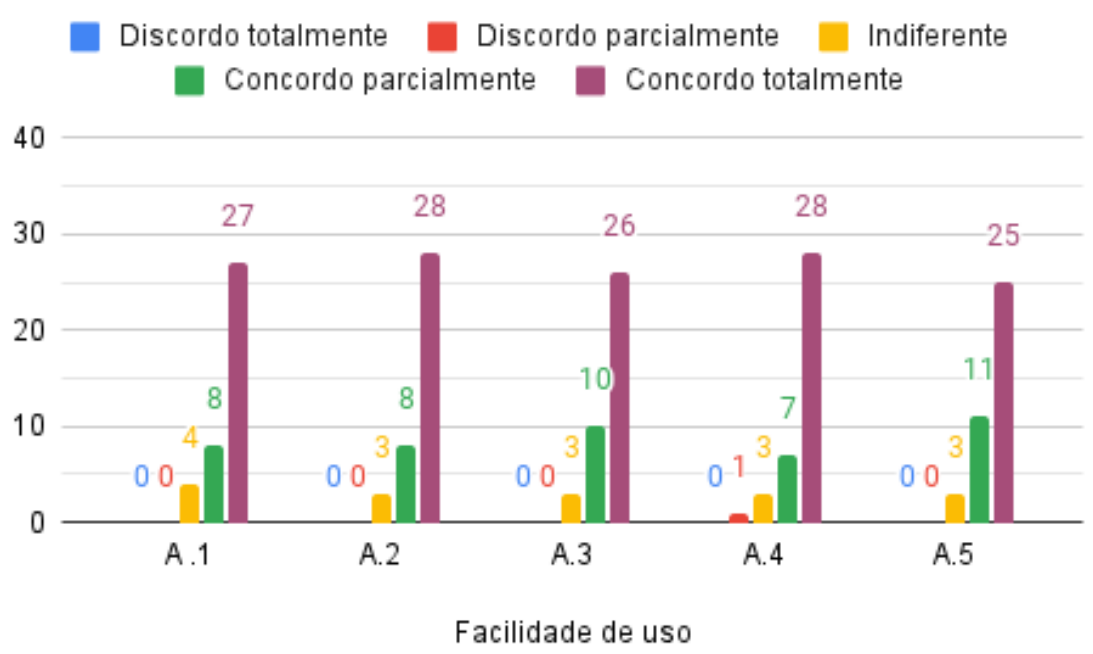

Figura 3. Questões sobre facilidade de Uso

aprender a utilizar o SofEMNo que acontecia durante a execução desse. Consequentemente, a maioria dos estudantes afirmaram que é fácil e intuitivo utilizá-lo. Apenas um usuário relatou discordar parcialmente sobre a facilidade de lembrar de como utilizar o software. Esse resultado pode ser justificado a partir de um comentário de um participante que afirmou que sem a descrição do cenário, dificilmente conseguiria preencher as informações solicitadas para a aplicação dos métodos.

\subsection{B - Utilidade do software}

A Figura 4 apresenta as percepções dos usuários quanto à utilidade do software para o aprendizado, bem como se ele melhoraria a produtividade e facilitaria a realização de atividades.

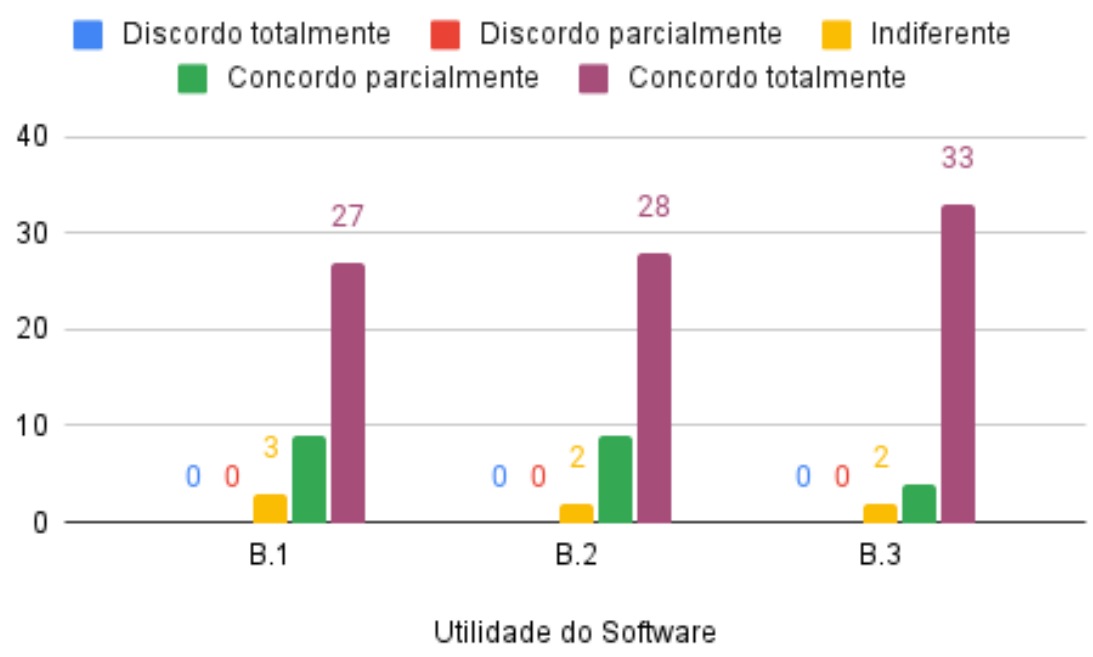

Figura 4. Questões sobre utilidade do software

Pela Figura 4, para 92.3\% (36) dos participantes, o software seria útil para melhorar o desempenho na disciplina de Métodos Numéricos e, além disso, 94.9\% (37) acredi- 
tam que estudar com o auxílio dessa ferramenta auxiliaria no aumento da produtividade para realização das atividades. Consequentemente, $94.9 \%$ (37) considera que o software facilitaria a realização das atividades.

Como observado na Figura 4, três participantes do teste classificaram como indiferente a utilidade do software para melhorar o aprendizado (pergunta B.1), dois que o software melhoraria a produtividade (B.2) e dois que o software facilitaria a realização das atividades (B.3). Entretanto nenhum participante rejeitou a ideia de utilização do software no processo de ensino e aprendizagem.

\subsection{C - Interface do software}

As questões apresentadas na Figura 5 tratam sobre a percepção em relação à interface do software, considerando aspectos como cores, fontes, botões, imagens, ícones, navegação e visualização de funcionalidades.

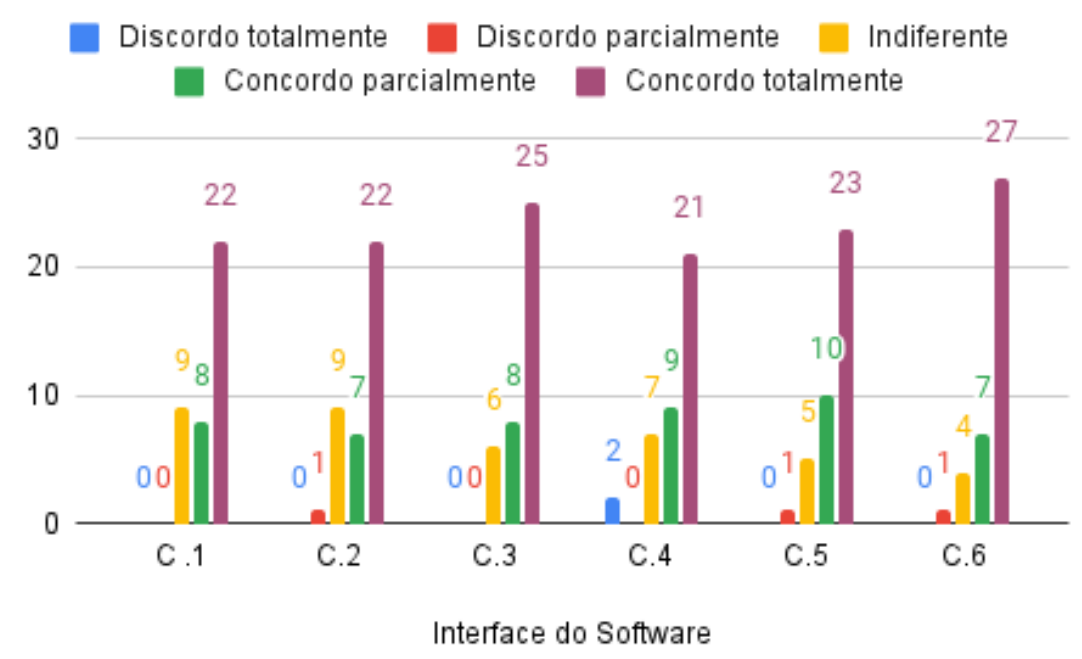

Figura 5. Questões sobre interface do software

$\mathrm{Na}$ Figura 5 verifica-se que a maior crítica foi com relação aos botões e fontes do software, uma vez que $76.9 \%$ (30) os consideraram agradáveis. Apenas uma resposta negativa (discordo parcialmente) foi observada com relação aos visualização dos botões e de informações dentro do software, como pode ser visto na questão C.2.

Ainda com relação ao visual do software, $76.9 \%$ (30) acharam que as imagens e ícones são de fácil reconhecimento. Além disso, 84.6\% (33) relataram entender com facilidade as palavras, nomenclaturas e ícones do software. Entretanto, dois participantes afirmaram terem tido dificuldade com os ícones do software (C.4).

Destaca-se que o software ainda encontra-se em desenvolvimento e o tamanho de fonte e botões devem ser incorporadas como melhorias, visto que já foi percebido pelos envolvidos no processo e agora confirmado com o teste de usabilidade.

\subsection{D - Ensino em sala de aula}

Por fim, a Figura 6 apresenta a percepção dos usuários com relação ao uso do software no ensino em sala de aula, verificando se ele facilitaria o ensino e aprendizagem, se tornaria 
as aulas mais interessantes, se ajudaria a explorar materiais, bem como se aumentaria o nível de aprendizagem e o domínio com as atividades de sala de aula.

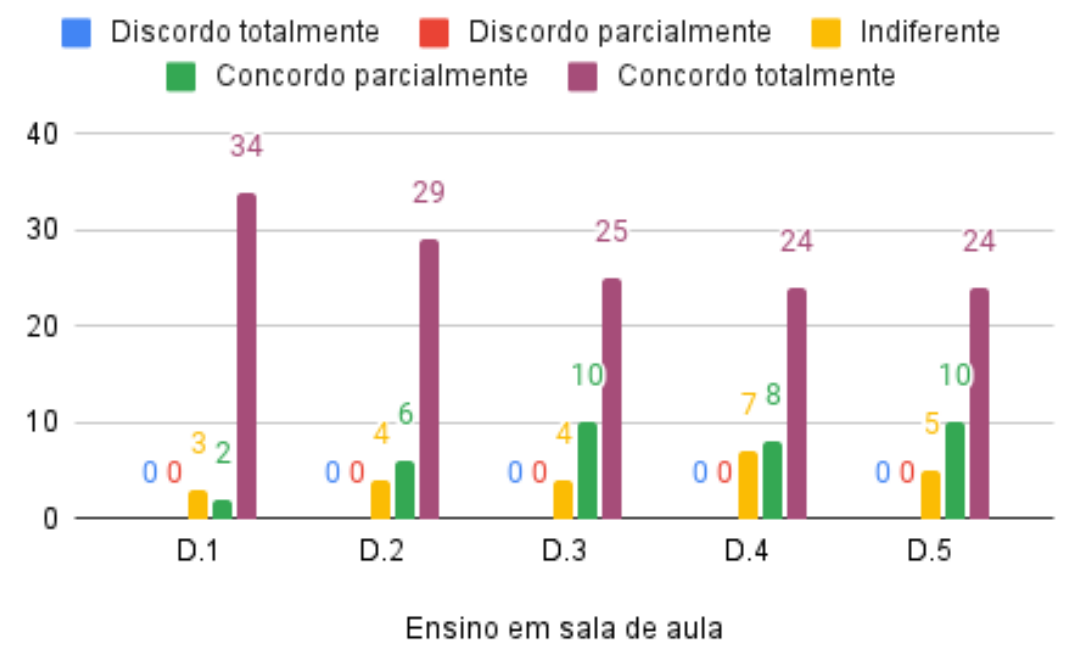

Figura 6. Questões sobre ensino em sala de aula

Na Figura 6, constata-se que nenhum participante rejeita o software como uma ferramenta que pode ser utilizada em sala de aula. Além disso, 89.7\% (35) consideram que com a utilização do SofEMN as aulas ficariam mais interessantes.

Também observa-se que $92.3 \%$ (36) dos alunos afirmaram que após se acostumar com a dinâmica do software, o ensino e a aprendizagem seriam facilitados. Para $89.7 \%$ (35) dos participantes, com o SofEMN, seria melhor explorar materiais disponíveis e $82 \%$ (32) dos alunos afirmam que teriam maior domínio sobre as atividades em sala de aula. Por fim, $87.1 \%$ (34) ressaltam que com o software o nível de aprendizagem aumentaria.

\subsection{Análise qualitativa}

O formulário de avaliação do software continha, além das questões objetivas, um espaço para registrar a "opinião sobre o software". A partir de uma análise de conteúdo foi possível perceber algumas características importantes descritas a seguir.

A maioria dos usuários consideraram o software intuitivo e muito interessante para conferir os resultados dos exercícios disponibilizados na disciplina de Métodos Numéricos. Salienta-se que quando a disciplina é desenvolvida solicita-se para os alunos o desenvolvimento de algoritmos e respectivas implementações em linguagens de programação, como C++ e Python, ou em softwares matemáticos, como Octave e MatLab. Tal fato contribui e justifica a opinião de todos sobre a "facilidade na conferência dos resultados dos exercícios". O software também foi considerado de fácil navegação, intuitivo, muito prático, amigável, facilitador para o entendimento dos processos e interessante para o aprendizado.

Verifica-se também que existe, pela grande maioria dos alunos, uma preocupação em utilizar o software para as atividades propostas na disciplina e facilitar a aprendizagem dos métodos numéricos, pois sugeriram que o software disponibilize os resultados parciais obtidos a cada iteração (passo a passo), o que contribuiria para o entendimento 
e conferência. Além disso, um aluno afirmou ter procurado ferramentas desse tipo para ajudá-lo nos estudos.

Foram sugestões de melhorias: realçar o display, aumentar as fontes, maior espaçamento entre os botões e disponibilizar uma janela de exibição maior. Por fim, muitos usuários solicitaram utilizar o software juntamente com o ensino dos métodos em sala de aula, o que tornaria a aula mais dinâmica e interessante.

Após perguntar se o usuário já havia utilizado algum outro software matemático, foi questionada a opinião dele comparando o SofEMN com o software já utilizado. A Figura 7 apresenta um resumo das respostas.

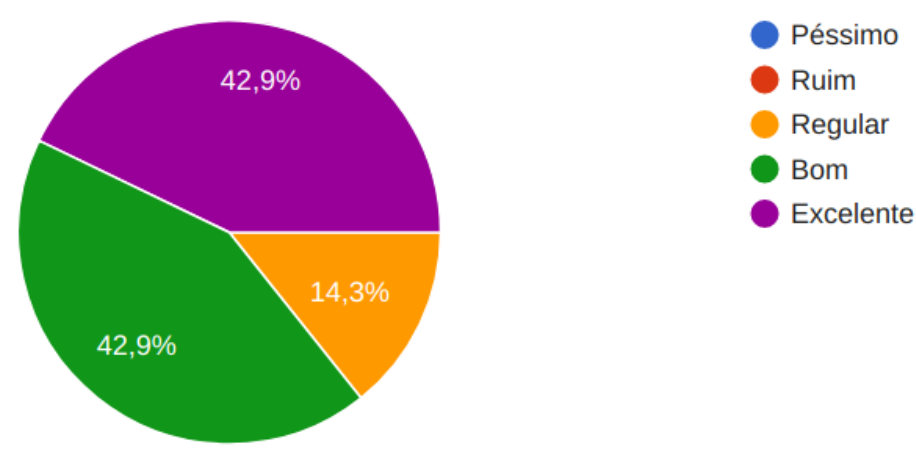

Figura 7. Comparação do SofEMN com outros softwares matemáticos

A maioria dos participantes afirmaram já terem utilizado algum software matemático, entre eles Matlab, Octave e SciLab. E que, em comparação a esses softwares, o SofEMN é uma excelente ferramenta.

É importante salientar que o SofEMN é um software livre específico para resolver problemas que necessitam de métodos numéricos na obtenção de soluções. Diferentemente dos softwares Matlab, Octave e SciLab que agregam uma ampla biblioteca matemática e que necessitam de implementações em seus ambientes.

\section{Considerações Finais}

Neste trabalho, a partir de uma primeira avaliação de usabilidade, foram realizadas análises qualitativas e quantitativas do SofEMN. O teste foi aplicado com 41 usuários, todos estudantes do ensino superior cursando a disciplina de Métodos Numéricos.

Um teste de usabilidade pode prover diferentes análises de um software. Neste artigo, o objetivo do teste foi verificar a facilidade de uso, utilidade, interface e uso em sala de aula de um software, em desenvolvimento, para auxílio no ensino e aprendizagem de métodos numéricos. A partir dos resultados percebe-se uma alta concordância com a facilidade em aprender e utilizar o software, bem como com a utilidade do software na realização de atividades e aprendizado de métodos numéricos.

Já em relação à interface, algumas sugestões de melhorias serão incorporadas ao software, tais como aumento da fonte dos textos e tamanho dos botões. Além disso, muitos sugeriram incluir o passo a passo da resolução, o que vai ao encontro de um software com foco educacional, proporcionando um feedback completo para o aluno. 
Com o objetivo de desenvolver um software educacional, é de suma importância o retorno dos usuários quando ao ensino em sala de aula. Os alunos demonstraram grande interesse em utilizar o software, sugerindo que seu uso seja adicionado ao escopo da disciplina, podendo aliar teoria e prática, além de facilitar a resolução de exercícios e verificação das respostas.

Como citado, esse foi o primeiro teste realizado com o SofEMN. Assim, como trabalhos futuros, cita-se a aplicação de testes com as demais funcionalidades desenvolvidas no software, já que, desde a aplicação do teste apresentado no presente trabalho, outras funcionalidades foram desenvolvidas e acrescentadas ao SofEMN, além de uma análise do desempenho dos alunos na disciplina com o uso do software.

Espera-se, juntamente com os futuros usuários da ferramenta, que seja possível melhorá-lo e torná-lo de grande valia no processo de ensino e aprendizagem.

\section{Referências}

Almeida, A., Gomes, T., Leal, V., Gomes, R., and Leal, L. (2018). Indicadores para avaliação de software educacional com base no guia gdsm (goal driven software measurement). Brazilian Symposium on Computers in Education (Simpósio Brasileiro de Informática na Educação - SBIE), 29(1):21.

Basili, V. and Rombach, H. (1988). The tame project: towards improvement-oriented software environments. IEEE Transactions on Software Engineering, 14(6):758-773.

Borges, V., Nogueira, B., and Barbosa, E. (2015). Uma análise exploratória de tópicos de pesquisa emergentes em informática na educação. Revista Brasileira de Informática na Educação, 23(01):85.

Chapra, S. C. (2013). Métodos numéricos aplicados com MATLAB para engenheiros e cientistas. AMGH, 3th edition.

Eaton, J. W. (2021). Gnu octave. In GNU Octave.

Likert, R. (1932). A technique for the measurement of attitudes. Archives of psychology.

Pires, P. S. M. (2004). Introdução ao scilab. In Scilab Versão 3.0. Manual. Federal do Rio Grande do Norte, Departamento de Engenharia de Computação e Automação.

Romero, C. S. (2006). Planejar aulas de matemática utilizando softwares educacionais. In Recursos Tecnológicos nas Instituições de Ensino.

Ruggiero, M. A. G. (1996). Cálculo Numérico: aspectos teóricos e computacionais. Makron Books, 2th edition.

Universidade Federal de Santa Maria-CS (2019). Projeto Pedagógico PPC - Engenharia Mecânica. Disponível em: https://www.ufsm.br/cursos/graduacao/cachoeira-dosul/engenharia-mecanica/projeto-pedagogico.

Valentim, N., Rabelo, J., Silva, W., Coutinho, W., Álvaro Mota, and Conte, T. (2014). Avaliando a qualidade de um aplicativo web móvel através de um teste de usabilidade: um relato de experiência. In Anais do XIII Simpósio Brasileiro de Qualidade de Software, pages 256-263, Porto Alegre, RS, Brasil. SBC. 